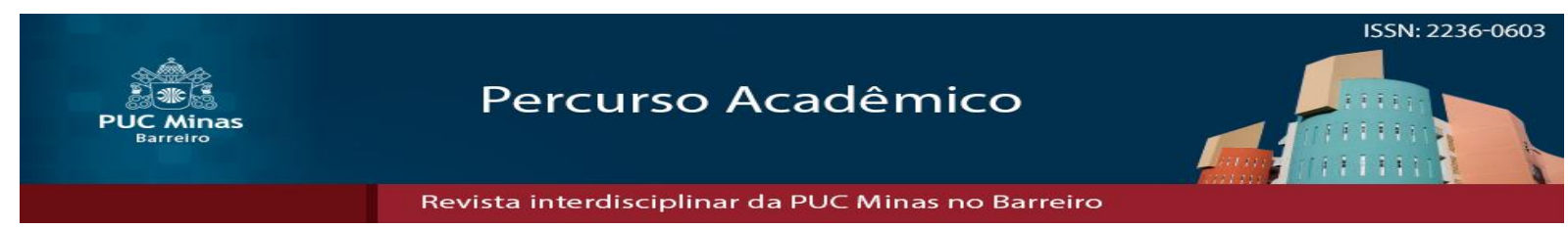

\title{
Avaliação da qualidade da refeição oferecida em uma Instituição de Longa Permanência
}

\section{Assessment of the quality of the meal offered to elderly residents in a long-stay institution}

\author{
Karoline Oliveira Abrantes ${ }^{1}$ \\ Luiza Vargas Mascarenhas Braga ${ }^{2}$ \\ Willian Fernandes de Oliveira ${ }^{3}$ \\ Márcia Regina Pereira Monteiro 4
}

\begin{abstract}
RESUMO
O presente trabalho teve como objetivo avaliar, de forma qualitativa e quantitativa, o cardápio oferecido durante três meses para as idosas residentes de uma Instituição de Longa Permanência para Idosos (ILPI), que fica localizada na cidade de Belo Horizonte - Minas Gerais. Para avaliar o cardápio utilizou-se o método de Avaliação Qualitativa de Preparações do Cardápio (AQPC), que é um método de avaliação global e permite uma análise geral das técnicas de cocção empregadas nas preparações. Já para avaliar aa aceitação dos cardápios oferecidos na ILPI, utilizou-se os cálculos de resto ingestão. Como resultado dessas análises foi possível observar um elevado valor de resto ingestão (obtendo-se média de 47,61g $\pm 24,68$ ), o que demonstrou a necessidade de uma reformulação dos cardápios, além da implementação de ações dentro da Unidade de Produção de Refeições (UPR) para que os cardápios sejam elaborados de modo a se adequar ao público, buscando-se maneiras para que as refeições cheguem à mesa com uma aparência mais atrativa. Sendo assim, tornam-se necessários alguns treinamentos e oficinas com as cuidadoras, cozinheiras e demais funcionários responsáveis pelo cuidado das idosas da ILPI, bem como a realização de um livro de receitas elaborado com as idosas, buscando solucionar os problemas encontrados na instituição.
\end{abstract}

Palavras-chave: Instituição de longa permanência para idosos. Capacitação em serviço. Alimentação coletiva. Unidade Produtora de Refeições. Qualidade da refeição. Avaliação do cardápio.

\footnotetext{
Artigo recebido em 31 de agosto de 2020 e aprovado em 13 de maio de 2021.

1 Graduanda em Nutrição pela Universidade Federal de Minas Gerais (UFMG), Brasil. E-mail: karolineabrantes@ hotmail.com

${ }^{2}$ Graduanda em Nutrição pela UFMG, Brasil. E-mail: luizavmb@gmail.com

${ }^{3}$ Graduado em Nutrição pela UFMG, Especialista em Terapia Nutricional e Doenças Crônicas pela Universidade Estadual Paulista (UNESP) Nutricionista Residente em Saúde do Adulto e Idoso na UNESP. E-mail: willianfbtu@ hotmail.com

${ }^{4}$ Doutorado em Ciência e Tecnologia de Alimentos pela Universidade Federal de Viçosa, mestrado em Agroquímica pela Universidade Federal de Viçosa, graduação em Nutrição pela Universidade Federal de Viçosa. Atualmente é professora associada do Departamento de Nutrição da Universidade Federal de Minas Gerais, Brasil. E-mail: mregina0@ hotmail.com
} 


\begin{abstract}
This study aimed to evaluate qualitatively and quantitatively, the menu used for three months in a home for elderly people in Belo Horizonte - MG. The menu evoluation was made by the qualitative method, this method allows to evaluate in general form and to analyze the culinary techniques of the preparations. To analysed the acceptance, calculation was used. The observed result was a high intake, showing the need to reformulate the menu according to the target audience, looking for new ways to make meals more attractive. Thus, it is necessary to promote training with nurses, cooks and other employees. As a result of these analyses it was possible to observe a high value of rest intake (averaging 47.61g \pm 24.68 ), which demonstrates the great need for a reformulation of menus, in addition to the implementation of actions within the Meal Production Unit so that the menus are designed to suit their audience.
\end{abstract}

Keys words: Long-stay institution for the elderly. Service training. Collective feeding. Meal Production Unit.

\title{
1 INTRODUÇÃO
}

Atualmente, nota-se a grande tendência de envelhecimento da população mundial. Em todo o mundo, a proporção da população com mais de 60 anos cresce de forma mais rápida do que a de outras faixas etárias, e a população de 80 anos ou mais é a que mais cresce, estimando-se que em 2050 ela passe dos atuais $11 \%$ para $19 \%$ o que sinaliza a necessidade de se conhecer mais sobre o envelhecimento, suas repercussões e impactos sobre o sistema de saúde brasileiro, sendo o envelhecimento sadio relacionado a uma boa nutrição (POLLO, 2008; CAMPOS, 2006). O estado nutricional adequado aumenta o número de pessoas que se aproximam do seu ciclo máximo de vida. É de extrema importância realizar a avaliação nutricional dos indivíduos idosos, pois através dela é possível identificar indivíduos em risco nutricional e, com isso, estabelecer programas de intervenção a fim de diminuí-los (CAMPOS, 2006; PEI et al., 2019).

A Pesquisa Nacional por Amostra de Domicílios Contínua (PNAD Contínua) de 2018 mostrou que o grupo das pessoas de 60 anos ou mais de idade, que representava 12,8\% da população residente total em 2012, passou para 15,4\% em 2018 (IBGE, 2018). O maior desafio no século XXI será cuidar dessa população crescente de idosos, grande parte com níveis socioeconômicos e educacionais baixos e com elevada prevalência de doenças crônicas e incapacitantes, devido a crescente demanda por procedimentos diagnósticos e terapêuticos de doenças crônicas não transmissíveis 
(DCNT), e a uma demanda ainda maior por serviços de reabilitação física e mental (LIMA, 2003).

Em termos nacionais, o Brasil tem apresentado um dos maiores crescimentos da população idosa e, além disso, a expectativa de vida dos brasileiros também aumentou de forma significativa (ALENCAR, 2012; MIRANDA et al, 2016). Porém, para muitos idosos, infelizmente, o aumento da longevidade vem junto de um declínio do estado de saúde físico e mental, além da presença de múltiplas doenças crônicas, da perda de independência e autonomia, e também de limitações socioeconômicas e ambientais, que são fatores associados à limitação da capacidade funcional desses idosos (ALENCAR, 2012; VERMA et al., 2020). Em decorrência da crescente demanda dos cuidados aos idosos, torna-se de extrema importância conhecer essa população, suas necessidades, hábitos e demandas, buscando assim, hábitos de vida saudáveis e a prevenção de doenças (CAMARGOS, 2015). O aumento da população idosa é uma realidade que exige modificar políticas públicas, estratégias sociais e econômicas, de modo a ter uma expectativa de vida saudável, reduzindo as deficiências físicas e as necessidades de cuidados especiais (SOUZA, 2016; VERAS e OLIVEIRA, 2018).

No Brasil, a legislação estabelece que os cuidados dos membros dependentes devam ser de responsabilidade de suas famílias, porém tais cuidados tem se mostrado cada vez mais insuficientes em virtude de várias mudanças como a redução da fecundidade, as mudanças nas taxas de casamento, e a crescente participação da mulher, tida como a principal cuidadora, no mercado de trabalho (CAMARANO, 2010).

Com todas essas mudanças ocorrendo na sociedade, tem se tornado crescente a demanda por Instituições de Longa Permanência para Idosos (ILPIs). As ILPIs são consideradas um sistema social organizacional, e têm como objetivo assistir ao idoso que não tenha vínculo familiar nem condições de manter o próprio sustento de modo a satisfazer as suas necessidades de moradia, alimentação, saúde e convivência social (CREUTZBERG, 2007). Segundo a Agência Nacional de Vigilância Sanitária (ANVISA), as ILPIs são “instituições governamentais ou não governamentais, de caráter residencial, destinada a domicilio coletivo de pessoas com idade igual ou superior a 60 anos, com ou sem suporte familiar, em condição de liberdade e dignidade e cidadania”, e a ANVISA também regulamenta a Resolução no 283 de 2005, que estabelece o regulamento técnico para o funcionamento das ILPIs, estabelecendo o padrão mínimo para o funcionamento dessas instituições (BRASIL, 2005). 
Algumas peculiaridades são inerentes ao processo do envelhecimento, como alterações orgânicas, fisiológicas, metabólicas, psicológicas e sociais. Essas características estão relacionadas à perda de apetite, menor percepção de sabor, menor sensibilidade olfativa e, em consequência disso, a menor ingestão de alimentos, o que pode levar o idoso à desnutrição. Além disso, algumas alterações no processo digestivo, na boca, estômago e intestino, podem ter como consequência a desnutrição (GALESI, 2009; YOUNG e MAGURI, 2019), alterando a qualidade de vida dessas pessoas. Essas disfunções sejam da percepção gustativa que constituem um problema relevante, a anomalia do paladar que afeta os efeitos diretos sobre a ingestão de alimentos e à contenção de um dos maiores prazeres da vida, o comer (KINA, 1998). Uma alimentação adequada e balanceada são condições para uma vida social, fisiológica e psicológica ideais (DE JESUS, 2017). Em relação aos idosos residentes em ILPIs, essas alterações podem ser aceleradas por agentes de ordem psicológica, seja a solidão provocada pelo isolamento social, em especial do meio familiar. Posto isso, faz-se importante a realização de trabalhos direcionados a este público com o intuito de promover uma melhoria na sua qualidade de vida, levando-se em consideração que a ILPI é uma questão pertinente para este público, e que são indispensáveis novos meios para melhorar a alimentação nas ILPIs, progredindo na qualidade de vida dos institucionalizados (JALES, 2005; CAMARGOS, 2015; DE JESUS, 2017).

Visto isso, é de extrema importância o papel da Unidade Produtora de Refeição (UPR) dentro de uma ILPI, já que um dos objetivos de uma UPR é o fornecimento de refeições que oferecem bom nível nutricional e sanitário e seja considerado de qualidade para o consumidor (PROENÇA, 1999). A UPR é direcionada para alimentação coletiva, como em instituições hospitalares e empresas, devendo contribuir ativamente para manter, melhorar e recuperar a saúde de seus comensais, através da alimentação equilibrada.

Em uma UPR, entende-se por qualidade a produção e o fornecimento de refeições nutricionalmente balanceadas e seguras do ponto de vista microbiológico (NOBRE, 2004), sendo função do nutricionista a preocupação e a garantia adequada nutricional da alimentação aos seus comensais, visando atender as necessidades, hábitos e preferências alimentares de seu público. Portanto, é essencial que dentro de uma ILPI o cardápio oferecido atenda às necessidades dos idosos residentes na instituição, fornecendo uma alimentação balanceada nutricionalmente e adequada qualitativamente, 
para que os idosos se alimentem de maneira correta, conforme abordado pela Portaria n 810, do Ministério da Saúde, sancionada em 1989, que estabelece as normas para o funcionamento de instituições geriátricas e destaca a responsabilidade da própria instituição nas produções de refeições para seus moradores (BRASIL, 2006).

Dessa forma, ressalta-se a importância de se resgatar a arte de cozinhar de forma saudável, planejando cardápios atentando para os aspectos nutricionais, higiênicos e sensoriais em uma ILPI, utilizando alimentos in natura e minimamente processados, como também, os métodos de preparo visando fornecer uma alimentação saudável para o consumidor final, os idosos.

O presente trabalho teve como objetivo avaliar, de forma qualitativa e quantitativa, o cardápio oferecido durante três meses para idosas residentes de uma Instituição de Longa Permanência (ILPI), localizada em Belo Horizonte - MG.

\section{METODOLOGIA}

Para realizar a avaliação dos cardápios oferecidos, optou-se por utilizar o método de Avaliação Qualitativa de Preparações do Cardápio (AQPC), que é um método de avaliação global e permite uma análise geral das técnicas de cocção empregadas nas preparações (VIEIROS, 2003). Já para realizar a avaliação da aceitação pelas idosas, exclusivamente do sexo feminino, dos cardápios oferecidos dentro da ILPI, utilizou-se o cálculo de resto ingestão.

Para este trabalho, foram avaliados os cardápios durante o período de três meses, entre os meses de abril e junho de 2018, e utilizou-se a metodologia sugerida por Proença em 2005, no qual as preparações são primeiramente divididas em quatro grupos: carnes, acompanhamentos - à base de arroz, massas, tubérculos e raízes, ou à base de legumes e verduras quentes - saladas e sobremesas (PROENÇA, 2005).

No momento de realizar a avaliação de aceitação pelas idosas dos cardápios oferecidos, utilizando os cálculos de resto-ingestão, utilizou-se os cálculos conforme descritos por VAZ (2006). Já para a análise da rejeição, com o valor de resto per capita obtido anteriormente, foi utilizado o teste de aceitabilidade, instituído pelo Ministério da Educação, conforme resolução No 26 de 17 de junho de 2013 (BRASIL, 2013), que dispõe sobre o atendimento da alimentação escolar aos alunos da educação básica no âmbito do Programa Nacional de Alimentação Escolar (PNAE) com a seguinte fórmula: 
Percentual de rejeição $(\%)=($ Peso da refeição rejeitada per capita $\mathrm{x} 100) /$ Peso da refeição distribuída per capita.

(BRASIL, 2013)

\section{RESULTADOS}

A avaliação dos cardápios da UPR da ILPI demonstrou boa rotatividade de alimentos, com técnicas de cocção distintas e alto valor nutricional, apresentando baixa presença de embutidos, mais de uma opção de salada, e sempre era oferecida uma fruta como sobremesa no almoço. Todos os dias, a refeição do jantar era a mesma oferecida no almoço, porém com acréscimo de sopa de legumes com macarrão. As outras refeições, como café da manhã e lanche da tarde, apresentavam um padrão: café com leite, café puro, leite puro, pão francês e pão doce com margarina e mingau. Vale ressaltar que as fichas técnicas dos cardápios apresentavam os procedimentos a que seriam realizados no dia seguinte, o que favorecia o trabalho para a realização das refeições.

A segunda avaliação realizada analisou o resto ingestão individual do almoço das idosas. Utilizaram-se a amostra de 44 idosas (91,6\% do total de residentes), todas do sexo feminino, com idade média de 80 anos. Durante sete dias não consecutivos foi observado o comportamento alimentar das mesmas no momento do almoço, e a refeição foi pesada em dois momentos: logo após se servir, e ao final da refeição. Durante a realização dos procedimentos, foi possível constatar que 27,3\% (n=12) das idosas consumiam dieta na forma pastosa, $47,7 \%(n=21)$ se alimentavam sentadas a mesa e $9,1 \%(\mathrm{n}=4)$ necessitavam do auxílio das cuidadoras para se alimentar, que as incentivava a comer tudo que era porcionado. Analisando os pratos de forma individual, encontrou-se uma média de 47,61 $\pm 24,68$ de sobra suja por idosa, e quando as sobras eram analisadas por dia, foi possível encontrar significativa diferença, mostrando assim a rejeição das idosas por preparações específicas, como pode ser visto no gráfico 1 abaixo. Na maior parte das sobras era encontrado carne. 
Gráfico 1: Resto da ingestão em gramas do almoço servido em uma Instituição de Longa Permanência em Belo Horizonte- MG, 2018.

Média e Desvio Padrão do Resto Ingestão do Almoço

Consumo de idosos em uma Instituição de Longa Permanência de Belo Horizonte (2018)

150

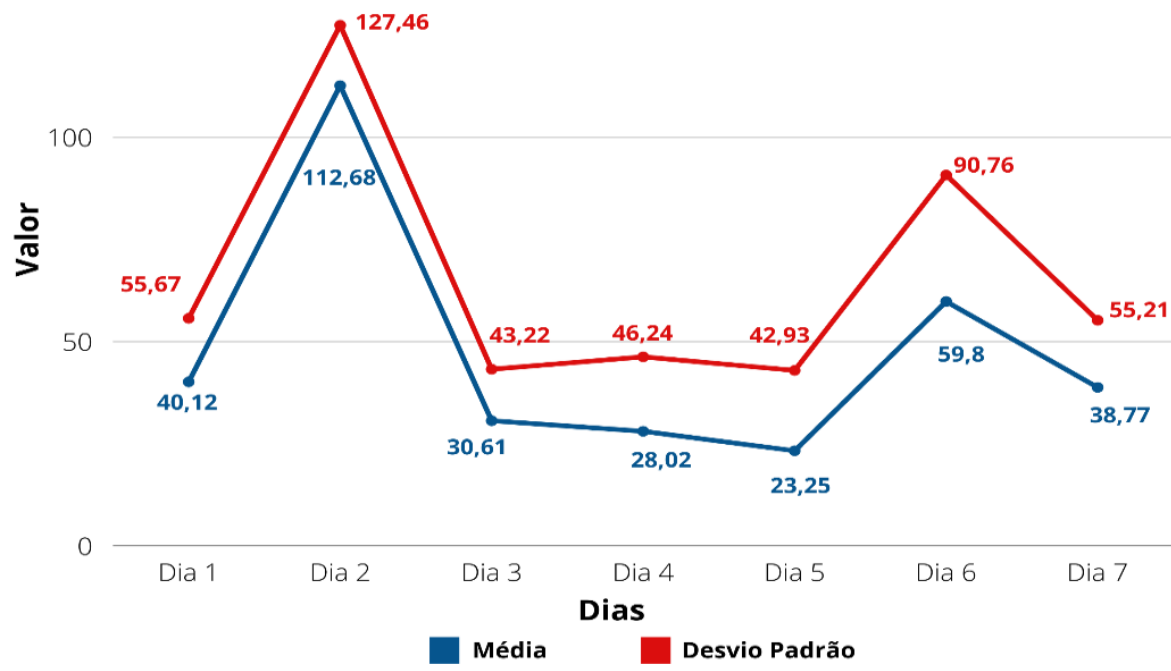

Fonte: Própria autoria

Além disso, as idosas que faziam suas refeições no refeitório durante o almoço apresentaram menor resto ingestão do que aquelas que não podiam descer e eram obrigadas a se alimentar no andar e com auxílio das cuidadoras.

\section{DISCUSSÃO}

Através das avaliações realizadas foi possível observar que o resto ingestão foi relativamente alto (Gráfico 1). Entretanto, de acordo com o que o resultado obtido durante o período em que foram realizadas as pesagens, grande parte desse resto ingestão poderia ser diminuído com medidas simples. Uma delas seria pela alteração do cardápio. O cardápio é uma ferramenta que traduz o trabalho realizado pelo nutricionista, e é por meio dele que se pode promover educação alimentar, saúde e melhor qualidade de vida (VEIROS, 2003). Devido à estagnação do cardápio e preferências pessoais, o consumo alimentar dos idosos tende a ser menor em relação ao que se é ofertado na ILPI. O obstáculo para ofertar o consumo alimentar adequado, ocorre no seu planejamento, levando em consideração as necessidades e preferências deste público (BROM, 2019). Um bom planejamento do cardápio proporciona aspectos nutricionais e sensoriais mais adequados, bem como permite uma avaliação anterior à execução dos pratos, sua composição e as preparações que o compõem. Verificam-se as técnicas de preparo, frequência dos alimentos, combinação de cores, permitindo fazermos uma análise ampla. Além disso, permite que seja alcançado o equilíbrio entre 
saudável e a aparência atrativa (CARVALHO, 2008; VIANA e MONTEIRO, 2016; RIBEIRO, 2019), como também, através dele, é possível adequá-lo com as características do público-alvo, no caso, os idosos, bem como adequar às propostas da UPR (RIBEIRO, 2019).

As idosas que não comiam à mesa, e sim sentadas em poltronas distribuídas nos andares, foram as que apresentaram maior taxa de resto ingestão, pois além de terem a distração da televisão no momento da refeição, ainda tinham de se adaptar para conseguir comer segurando o prato nas mãos, sem nenhum apoio. Muitas vezes alimentos caíam no chão e no colo das idosas, demonstrando a grande dificuldade que elas tinham ao se alimentar, muitas delas apresentando falta de dentição. O envelhecimento pode também predeterminar a alterações fisiológicas e patológicas na dentição, tendo assim interesse em abordar a influência dessas particularidades do idoso na escolha da conduta mais adequada ao tratamento (RIVALDO, 2008; BEZERRA, 2012). Ainda, observou-se também que o resto ingestão variava de acordo com o cardápio ofertado no dia. Às sextas feiras, quando a fonte proteica oferecida era ovo, e não carne, o resto ingestão se mostrou maior do que nos outros dias analisados.

Como relatado, as idosas com menor capacidade cognitiva e motora, não desciam para o refeitório para realizar as refeições, sendo as cuidadoras as responsáveis por servir a alimentação dessas idosas no próprio dormitório, o que muitas vezes fazia com que a quantidade de alimento oferecida para elas não estivesse de acordo com o apetite delas naquele momento. Além disso, as cuidadoras que deduziam quais alimentos as idosas desejam ou não comer, não dando a elas a oportunidade de observar e escolher os próprios alimentos. Tal prática acaba aumentando o resto ingestão e, muitas das vezes, fazendo com que a idosa não coma algum alimento não por não gostar, mas sim por este não ser ofertado a ela.

Monteiro et al. 2015, realizou um estudo em uma ILPI também localizada na cidade Belo Horizonte MG, com 30 idosos residentes, sendo 77\% ( $\mathrm{n}=23)$ dos idosos eram do sexo feminino, e 23\% (n=7) masculino e mostrou que há inadequação no consumo em comparação com as recomendações diárias desses idosos (MONTEIRO, 2015). Brom et al., 2019, também realizou um estudo em 8 ILPIs localizadas em Belo Horizonte e Contagem, Minas Gerais e observaram que em apenas duas instituições das oito atingiram a recomendação esperada de $1873 \mathrm{kcal}$ para mulheres, e apenas uma atingiu a recomendação de 2054 kcal para homens, com valor energético médio das 
refeições (1657 kcal $\pm 372,58 \mathrm{kcal})$, demonstrando uma oferta insuficiente em $75 \%$ das ILPI para mulheres e em 87,5\% para homens. Nas instituições em que havia nutricionista, o resultado foi adequado $(108 \%$ e $91 \%)$, nas outras que não haviam, a adequação foi bem inferior (69\%) (BROM, 2019). Em uma análise do cardápio de uma ILPI do Município do Recôncavo da Bahia mostrou que não houve oferta de folhosos, baixa coloração no prato e alta oferta de doces (SANTANA, 2019), diferente do recomendando pelo Guia Alimentar para a População Brasileira 2014 (BRASIL, 2014). Estes estudos apresentam resultados de inadequação no consumo, assim como o nosso.

Diante dos resultados, foi elaborado um livro de receitas com a participação das idosas. O objetivo deste livro foi proposto com o intuito de resgatar os hábitos alimentares saudáveis, através da memória das idosas e das receitas que elas e suas famílias executavam durante o convívio familiar. O livro foi elaborado envolvendo três etapas. Na primeira etapa foram realizadas entrevistas individuais com relatos das idosas. Na segunda etapa foi realizada a construção do livro de receitas através dos relatos das idosas. Na terceira etapa, a execução das receitas com as idosas. Ao todo foram coletadas 21 receitas. Este livro será disponibilizado na ILPI, a fim de que se possa introduzir essas receitas no cardápio da instituição. Mas também, deixar registrado um pouco da memória de cada uma, com o intuito das mesmas conversarem sobre as receitas que mais marcaram entre elas, tornando a instituição um local em que as idosas possam se sentir mais "em casa”. Foi possível notar, através das entrevistas, a importância da relação das idosas e suas mães na construção das receitas, pois todas as entrevistadas enfatizaram os laços maternos ao descreverem as receitas.

Dessa forma, torna-se necessário avaliar a viabilidade de se aplicar alguns treinamentos e oficinas com as cuidadoras e as cozinheiras da instituição, buscando reduzir e/ou minimizar os problemas observados, bem como a importância do nutricionista dentro da ILPI. As intervenções teriam como foco explicar a importância do momento da refeição e também da importância da redução do resto ingestão, focando em dois fatores principais: a nutrição das idosas residentes da instituição e também na redução do desperdício de alimentos.

\section{CONSIDERAÇÕES FINAIS}

A partir dos resultados encontrados, foi possível perceber que se faz necessário uma melhora no planejamento dos cardápios com preparações que atendam as 
preferências e hábitos alimentares das idosas residentes da ILPI, uma vez que os índices de resto ingestão se mostraram altos. Possibilitou conhecer a realidade de uma ILPI, os desafios e dificuldades encontrados e a rotina enfrentada pelos profissionais que dão suporte, apoio e assistência no cuidado das idosas. Também serão propostas algumas oficinas com as cozinheiras, as cuidadoras, e com as próprias idosas, com a intenção de tornar a alimentação e o momento de seu preparo algo mais prazeroso para todos os envolvidos.

\section{REFERÊNCIAS}

ALENCAR, Mariana Asmar et al. Perfil dos idosos residentes em uma instituição de longa permanência. Revista Brasileira de Geriatria e Gerontologia, v. 15, n. 4, p. 785-796, 2012. Disponível em: < https://www.scielo.br/scielo.php?pid=S1809$98232012000400017 \&$ script=sci_abstract\&tlng=pt $>$

BEZERRA, Fernanda Carvalho; ALMEIDA, Maria Irismar de; NÓBREGATHERRIEN, Sílvia Maria. Estudos sobre envelhecimento no Brasil: revisão bibliográfica. Revista brasileira de geriatria e gerontologia, v. 15, n. 1, p. 155-167, 2012. Disponível em: < https://www.scielo.br/scielo.php?pid=S1809$8232012000100017 \&$ script=sci_abstract\&tlng=pt $>$

BRASIL. MINISTÉRIO DA SAÚDE. Guia alimentar para a população brasileira. Ministério da Saúde, 2014. Disponível em: < https://bvsms.saude.gov.br/bvs/publicacoes/guia_alimentar_para_a_pop_brasiliera_miol o_internet.pdf>

BRASIL. MINISTÉRIO DA SAÚDE. Secretaria de Atenção à Saúde. Departamento de Atenção Básica. Cadernos de Atenção Básica: Envelhecimento e saúde da pessoa idosa. Ministério da Saúde, Secretaria de Atenção à Saúde. Departamento de Atenção Básica. Brasília: Ministério da Saúde, 2006. Disponível em: < https://bvsms.saude.gov.br/bvs/publicacoes/evelhecimento_saude_pessoa_idosa.pdf>

BRASIL. Resolução/CD/FNDE n 26 , de 17 de junho de 2013. Dispõe sobre o atendimento da alimentação escolar aos alunos da educação básica no âmbito do Programa Nacional de Alimentação Escolar (PNAE). Diário Oficial da União, 2013. Disponível em: < https://www.fnde.gov.br/acesso-ainformacao/institucional/legislacao/item/4620-resolu\%C3\%A7\%C3\%A3o-cd-fnden\%C2\%BA-26,-de-17-de-junho-de-2013>

BROM, Izabella Ferreira Gomes Carneiro et al. Avaliação da composição nutricional de cardápios em instituições de longa permanência para idosos em Belo Horizonte e Contagem, Minas Gerais. Revista da Associação Brasileira de Nutrição-RASBRAN, v. 10, n. 1, p. 87-95, 2019. Disponível em: < https://www.rasbran.com.br/rasbran/article/view/1286/246> 
CAMARANO, Ana Amélia; KANSO, Solange. As instituições de longa permanência para idosos no Brasil. Revista Brasileira de Estudos de População, v. 27, n. 1, p. 232235, 2010. Disponível em: < https://www.scielo.br/pdf/rbepop/v27n1/14.pdf>

CAMARGOS, Mirela Castro Santos et al. Aspectos relacionados à alimentação em Instituições de Longa Permanência para Idosos em Minas Gerais. Cadernos Saúde Coletiva, v. 23, n. 1, p. 38-43, 2015. Disponível em: < https://www.scielo.br/scielo.php?pid=S1414462X2015000100038\&script=sci_abstract\&tlng=pt>

CAMPOS, Marta Alice Gomes et al. Estado nutricional e fatores associados em idosos. Revista da Associação Médica Brasileira, v. 52, n. 4, p. 214-221, 2006. Disponível em: < https://www.scielo.br/scielo.php?pid=S010442302006000400019\&script=sci_arttext\&tlng=pt >

CARVALHO, Luciana Carvalho de. Avaliação qualitativa das preparações do Festival Brasil Sabor Brasília: festival gastronômico de 2007- 2008. 58 f. Monografia (Especialização em Gastronomia e Saúde)- Universidade de Brasília, Brasília, 2008. Disponível em: < https://bdm.unb.br/bitstream/10483/333/1/2008_LucianaCarvalhoCarvalho.pdf>

CREUTZBERG, Marion et al. A instituição de longa permanência para idosos e o sistema de saúde. Revista Latino-americana de Enfermagem, v. 15, n. 6, p. 11441149, 2007. Disponível em: < https://www.scielo.br/scielo.php?script=sci_arttext\&pid=S0104$11692007000600014 \& \operatorname{lng}=$ pt\&nrm=iso\&tlng=en >

DE JESUS, Miriam Aparecida Taver; SZCZEREPA, Sunáli Batistel. Composição nutricional do cardápio de uma instituição de longa permanência de idosos maiores de 70 anos em Ponta Grossa/PR. Revista da Associação Brasileira de NutriçãoRASBRAN, v. 8, n. 2, p. 27-36, 2017. Disponível em: < https://www.rasbran.com.br/rasbran/article/view/519/184>

GALESI, Lilian Fernanda et al. Perfil alimentar e nutricional de idosos residentes em moradias individuais numa instituição de longa permanência no leste do estado de São Paulo. Alimentos e Nutrição Araraquara, v. 19, n. 3, p. 283-290, 2009. Disponível em: < Perfil alimentar e nutricional de idosos residentes em moradias individuais numa instituição de longa permanência no leste do estado de São Paulo>

INSTITUTO BRASILEIRO DE GEOGRAFIA E ESTATÍSTICA - IBGE. Pesquisa Nacional por Amostra de Domicílios Contínua: características gerais dos domicílios e dos moradores 2017. Rio de Janeiro: IBGE, 2018. Disponível em: <<https://biblioteca.ibge.gov.br/visualizacao/livros/liv101566_informativo.pdf > Acesso em: 06 de Março de 2020.

JALES, Mariana Amorim et al. Características do sistema estomatognático em idosos: diferenças entre instituição pública e privada. Revista CEFAC, v. 7, n. 2, p. 178-187, 2005. Disponível em: < https://www.redalyc.org/articulo.oa?id=169320502005> 
KINA, Sidney et al. Alterações da sensibilidade gustativa no paciente idoso. Atualidades em Geriatria, v. 3, n. 18, p. 20-22, 1998.

LIMA-COSTA, Maria Fernanda; VERAS, Renato. Saúde pública e envelhecimento. Cadernos de Saúde Pública, v. 19, n. 3, p. 700-701, 2003. Disponível em: < https://www.scielo.br/scielo.php?script=sci_arttext\&pid=S0102311X2003000300001>

\section{MINISTÉRIO DA SAÚDE. RESOLUÇÃO - RDC No 283, DE 26 DE SETEMBRO} DE 2005, 2005. Disponível em: <http://portal.anvisa.gov.br/documents/10181/2718376/RDC_283_2005_COMP.pdf/a3 8f2055-c23a-4eca-94ed-76fa43acb1df> Acesso em: 06 de Março de 2020.

MIRANDA, Gabriella Morais Duarte; MENDES, Antonio da Cruz Gouveia; SILVA, Ana Lucia Andrade da. Population aging in Brazil: current and future social challenges and consequences. Revista Brasileira de Geriatria e Gerontologia, 19(3), 507-519. 2016. https://dx.doi.org/10.1590/1809-98232016019.150140

MONTEIRO, Marlene Azevedo Magalhães; MAIA, Isabel Cristina Miranda Pinheiro. Perfil Alimentar De Idosos Em Uma Instituição De Longa Permanência De Belo Horizonte, Minas Gerais. Revista de APS, v. 18, n. 2, 2015. Disponível em: < https://periodicos.ufjf.br/index.php/aps/article/view/15519>

NOBRE, Andreza Paulo. Avaliação da satisfação da clientela em restaurantes do tipo self-service de Brasília.37 f. Monografia (Especialização em Qualidade em Alimentos)- Universidade de Brasília, Brasília, 2004. Disponível em: < https://bdm.unb.br/bitstream/10483/488/1/2004_AndrezaPauloNobre.pdf>

POLLO, Sandra Helena Lima; ASSIS, Mônica de. Instituições de longa permanência para idosos-ILPIS: desafios e alternativas no município do Rio de Janeiro. Revista Brasileira de Geriatria e Gerontologia, v. 11, n. 1, p. 29-44, 2008. Disponível em: < https://www.scielo.br/scielo.php?script=sci_arttext\&pid=S1809-98232008000100029>

PROENÇA, Rossana Pacheco da Costa. Novas tecnologias para a produção de refeições coletivas: recomendações de introdução para a realidade brasileira. Revista de Nutrição, v. 12, n. 1, p. 43-53, 1999. Disponível em: < https://www.scielo.br/pdf/rn/v12n1/v12n1a04.pdf>

PROENÇA, Rossana Pacheco da Costa. Qualidade nutricional e sensorial na produção de refeições. p. 221-221, 2005.

RIBEIRO, Rita de Cássia. Cardápios: Uma nova abordagem para o diagnóstico, planejamento e avaliação. 1. ed. Minas Gerais: UFMG. 106 p. v. 1. ISBN $9788542302950,2019$.

RIVALDO, Elken Gomes et al. Envelhecimento e saúde bucal. Stomatos, v. 14, n. 26, p. 39-45, 2008. Disponível em: < https://www.redalyc.org/pdf/850/85012264006.pdf>

SANTANA, Bianca de Souza. Alimentação do idoso: análise do cardápio de uma instituição de longa permanência em um município do Recôncavo da Bahia. 2019. 
Karoline Oliveira Abrantes, Luiza Vargas Mascarenhas Braga, Willian Fernandes de Oliveira e Márcia Regina Pereira Monteiro

SOUZA, Marilza Amaral Henrique de et al. Perfil do estilo de vida de longevos. Revista Brasileira de Geriatria e Gerontologia, v. 19, n. 5, p. 819-826, 2016. Disponível em: < https://www.scielo.br/pdf/rbgg/v19n5/pt_1809-9823-rbgg-1905-00819.pdf>

VAZ, Célia Silvério. Restaurantes: controlando custos e aumentando lucros. Brasília: Metha, p. 196, 2006.

VEIROS, Marcela Boro; PROENÇA, Rossana PC. Avaliação qualitativa das preparações do cardápio em uma unidade de alimentação e nutrição-método AQPC. Nutrição em Pauta, v. 11, n. 62, p. 36-42, 2003. Disponível em: <https://www.nutricaoempauta.com.br/lista_artigo.php?cod=108>

VERAS, Renato Peixoto; OLIVEIRA, Martha. Envelhecer no Brasil: a construção de um modelo de cuidado. Ciênc. saúde coletiva, Rio de Janeiro, v. 23, n. 6, p. 19291936, jun. 2018. Disponível em <http://www.scielo.br/scielo.php?script=sci_arttext\&pid=S1413$81232018000601929 \& \operatorname{lng}=\mathrm{pt} \& \mathrm{nrm}=\mathrm{iso}>$. Acesso em: 25 ago. 2020. http://dx.doi.org/10.1590/1413-81232018236.04722018

VERMA, Madhur et al. Screening for cognitive impairment among the elderly attending the noncommunicable diseases clinics in a rural area of Punjab, North India. Asian Journal of Psychiatry. 2020. DOI://doi.org/10.1016/j.ajp.2020.102001

VIANA, M.B, MONTEIRO, M.R.P. Avaliação Qualitativa das Preparações do Cardápio em uma unidade produtora de refeições de Belo Horizonte/MG. Revista Nutrição Brasil, v.15, n.1, 2016. Disponível em: <https://portalatlanticaeditora.com.br/index.php/nutricaobrasil/article/view/106/0>

YOUNG, Frances; MAGUIRE, Simon. Physiology of ageing. Anaesthesia Journal and Intensive Care Medicine, v. 20, p 735-738. 2019. DOI:

https://doi.org/10.1016/j.mpaic.2019.10.006 